\title{
Artes escénicas, nuevas tecnologías, patrimonio museístico y su aplicación como herramienta de cambio social con un grupo de víctimas de violencia y explotación sexual
}

\author{
Amador Cernuda-Lago ${ }^{1}$
}

Recibido: 26 de agosto de 2019 / Aceptado: 2 de septiembre de 2019 / Publicado: 15 de octubre de 2019

Resumen. Estudio de los beneficios del Arte en intervención clínica y social mediante propuestas de educación artística patrimonial en museos, actividades de arteterapia escénica, psicoterapia cognitiva con apoyo en nuevas tecnologías como la realidad virtual. Método: 34 mujeres, víctimas de explotación y violencia sexual, se entrevistaron clínicamente y evaluaron con test psicológicos. 48 sesiones de psicoballet y arteterapia escénica, 8 visitas guiadas a museos de arte y etnología y 24 sesiones de nuevas tecnologías de apoyo a la psicoterapia cognitiva y a la sensibilización artística patrimonial. Resultados: descendió la ansiedad un $40 \%$. Conclusiones: la intervención mediante las artes ha permitido una innovación en la recuperación alternativa a la palabra.

Palabras clave: Prostitución; rehabilitación; arteterapia; realidad virtual; patrimonio.

[en] Performing Arts, New Technologies, Museum Heritage and its Application as a Tool for Social Change with a Group of Victims of Violence and Sexual Explotation

\begin{abstract}
This article studies the benefits of Art in clinical and social intervention through proposals of heritage artistic education in museums, scenic art activities, cognitive psychotherapy with support in new technologies such as virtual reality. Method: 34 women, victims of exploitation and sexual violence, were interviewed clinically and evaluated with psychological tests. 48 sessions of psychoballet and scenic art therapy, 8 guided visits to art and ethnology museums and 24 sessions of new technologies to support cognitive psychotherapy and heritage artistic awareness. Results: anxiety decreased $40 \%$, increasing self-esteem $35 \%$. Conclusions: intervention through the arts has allowed an innovation in alternative recovery to the word.
\end{abstract}

Keywords: Prostitution; Rehabilitation; Art Therapy; Virtual Reality; Heritage.

Sumario. 1. Introducción. 2. Prostitución y arte. 3. Metodología. 3.1. Sujetos. 3.2. Instrumentos. 3.3. Procedimiento. 4. Resultados. 5. Discusión. 6. Conclusiones. 7. Fuentes y referencias bibliográficas.

Cómo citar: Cernuda-Lago, Amador. "Artes escénicas, nuevas tecnologías, patrimonio museístico y su aplicación como herramienta de cambio social con un grupo de víctimas de violencia y explotación sexual". En Museo. Imagen. Sentidos, editado por Ángel Pazos-López y Alejandra Alonso Tak. Monográfico temático, Eikón Imago 14 (2019): 73-87.

1 Universidad Rey Juan Carlos.

Correo electrónico: amador.cernuda@urjc.es

ORCID: https://orcid.org/0000-0002-5993-4647 


\section{Introducción}

La experiencia de haber llevado a cabo varios programas de apoyo y rehabilitación de víctimas de explotación sexual nos ha permitido observar que, además de la dificultad lógica esperable de los procesos, uno de los aspectos más destacable y que complica la rehabilitación es la marcada incidencia de patología dual. Hemos detectado mucha comorbilidad de adicción a drogas y alcohol, con problemas de autoestima, ansiedad y depresión ${ }^{2}$. Después de analizar los datos de 3 programas de intervención realizados en los últimos 5 años hemos detectado una serie de variables presentes en todas las mujeres que han logrado superar su situación de explotación, que han podido salir de ese ambiente y que han reiniciado una vida alternativa totalmente al margen de su vida anterior, encontrando que, incluso para salir de la marginación, es importante tener inteligencia emocional. La realidad de la prostitución abarca múltiples contextos y resulta compleja.

"Los datos ofrecidos por la Organización Mundial de la Salud (OMS) ${ }^{3}$ y diversas organizaciones defensoras de los derechos humanos indican que, tanto en nuestro país como en toda la Unión Europea, existen distintos tipos de prostitución con características y problemáticas diversas: más del $90 \%$ proviene del tráfico sexual migratorio, controlado por las mafias del sexo, que mantienen a las mujeres en condiciones infrahumanas, y cada año incorporan unas 500.000 nuevas esclavas sexuales; en el otro extremo, una minoría que no alcanza el $1 \%$, pertenece a lo que se llama prostitución de lujo, con un alto nivel de vida y de posibilidades. Sólo alrededor de un 5\% afirma que ha elegido libremente esta actividad porque le gusta y lo considera un trabajo como otro cualquiera. El resto de las prostitutas, el 95\%, argumenta que lo hace por necesidad, porque no tiene otra salida para ganarse la vida y afirma que no le gusta ni lo quiere para sus hijas. Sólo una minoría ejerce como autónoma, mientras que la inmensa mayoría está controlada por las mafias, empresarios y otros proxenetas"."

El presente trabajo se centra en experiencias relacionadas con la prostitución de mujeres inmigrantes y víctimas de explotación sexual. No atiende a otros colectivos como transexuales, prostitución masculina o a otras problemáticas como la prostitución infantil o a las lamentables situaciones que acontecen en países en vías de desarrollo.

El trabajo diario con mujeres que ejercen la prostitución nos demuestra que muchas están expuestas constantemente a múltiples acontecimientos estresantes en su vida diaria: situación de ilegalidad, miedo constante a ser detenidas y expulsadas del país, miedo a personas que les importunan en las calles, pago de deudas como

2 Amador Cernuda, "Efectos de un programa de rehabilitación psicosocial y educativa mediante actividad física y artística con un grupo de víctimas de explotación sexual", en III Congreso Internacional Multidisciplinar de Investigación Educativa-CIMIE14, 3-4 de julio de 2014, Segovia, consultado el 28 de marzo de 2019, http://amieedu.org/actascimie14/?cat $=18$

3 Organización Panamericana de la Salud, "Trata de personas. Comprender y abordar la violencia contra las mujeres", OMS: WHO/RHR/12.42, 2013, consultado el 28 de marzo de 2019, https://www.who.int/reproductivehealth/publications/violence/rhr12_42/es/

$4 \quad$ Soledad Muruaga, "Prostitución y salud", AMS mujeres para la salud, consultado el 28 de marzo de 2019, https://www.mujeresparalasalud.org/prostitucion-y-salud/ 
se da el caso en mujeres inmigrantes que han sido manipuladas por mafias, miedo a la posibilidad de elegir un cliente que pueda hacerles daño, tener que utilizar varios nombres y varios estilos de vida para evitar ser excluidas por la sociedad, etc., todas estas circunstancias confieren un clima extremo en su proceso vital, máxime si, además de los posibles desencadenantes que conducen a esta actividad, las personas involucradas son víctimas de explotación y en realidad de verdadera esclavitud manejada por grupos mafiosos y criminales.

Para cada mujer el ejercicio de la prostitución representa un significado único, muchas de ellas comparten la visión de que es una actividad degradante y que sólo se realiza por necesidad económica o por incapacidad de acceder a un puesto de trabajo normalizado, encontrándonos con dos situaciones: mujeres que no consiguen adaptarse al tipo de vida y al hecho de prostituirse, pero que se mantienen en ella porque no tienen otra opción -presión de una deuda, que es un aspecto generalizado en el caso de víctimas de explotación- y, en segundo lugar, mujeres que consideran el ejercicio de la prostitución como algo temporal y circunstancial y que son capaces de proyectarse al futuro, un futuro que ya no tiene nada que ver con el mundo de la prostitución, son mujeres que se identifican con lo que hacen y no tiene ningún planteamiento de abandono del ejercicio de la prostitución y que mantienen un nivel de autoestima significativo, pero hay que señalar que esta situación es minoritaria. Por tanto, podemos afirmar que las mujeres que ejercen la prostitución conforman un grupo muy heterogéneo. Categorizar un perfil es difícil ya que existen muchos criterios y múltiples definiciones sobre el fenómeno de la prostitución. La generalización de algunos estereotipos y concepciones erróneas sobre este colectivo han interferido en todos los aspectos relacionados con el tema, como son las políticas de intervención y de investigación y por ello el abordaje del problema ha sido muy confuso.

Con respecto a la investigación sobre el colectivo de personas que ejercen la prostitución se ha podido constatar una evolución a lo largo del siglo XX y, en especial, en la década de los 80 y 90 la literatura científica ha incrementado los estudios sobre este colectivo; sin embargo, es necesario señalar las carencias o problemas detectados en tales estudios, como señala Vanwesenbeeck ${ }^{5}$ : por un lado, nos encontramos con estudios parciales que se centran en aspectos muy concretos (prostitución-VIH-Sida, prostitución-Infecciones de Transmisión Sexual, prostitución-trastornos mentales asociados, etc.) y no ofrecen una visión global de todas las variables que inciden en la salud y bienestar del colectivo (variables personales, sociales, sanitarias, psicológicas, económicas) y por otro, son estudios con poblaciones muy concretas y específicas, los datos no pueden ser generalizables a todos los tipos de prostitución (de calle, pisos, club, etc.), ni a los diferentes colectivos de personas que ejercen la prostitución (mujeres, hombres, transexuales), o a las diferentes zonas geográficas (zonas urbanas, rurales, sociedades occidentales, no occidentales, países del primer mundo, del tercer mundo).

Los estudios más recientes sobre la "calidad de vida" y la "salud física" de las personas que ejercen la prostitución analizan los aspectos más negativos y

Ine Vanwesenbeeck, "Another decade of social scientific work on sex work: A review of research 19902000”, Annual Review of Sex Research 12 (2001): 242-289, https://www.ncbi.nlm.nih.gov/pubmed/12666742 
estigmatizantes. En cualquier revisión bibliográfica que realicemos en el Medline con el término "prostitution" y "health" aparecen más de dos mil citas, en su mayoría referidas al VIH-Sida, a las infecciones y enfermedades de transmisión sexual (ITS o ETS) y al consumo de drogas.

La novedad que aportan los estudios de la última década sobre prostitución de calle y drogas es que no se centran tanto en la constatación sobre la prevalencia de las enfermedades, sino que intentan analizar otros factores que pueden mejorar su calidad de vida:

Un estudio sobre la evaluación de las necesidades de salud de las mujeres que ejercen en la calle en Bristol ${ }^{6}$. Los autores aplicaron una entrevista a 70 mujeres y todas informaron tener problemas de salud crónicos. Las ITS eran entre 9 y 60 veces más comunes que en la población general. Muchas mujeres (44 \%) habían experimentado abuso sexual y el $38 \%$ por ciento había recibido atención. La mayoría $(97 \%)$ habían recibido más dinero para tener relaciones sexuales sin protección. La mitad (51\%) tenía relaciones sexuales sin protección. Todas tenían problemas de drogas o dependencia del alcohol. En la última semana, el $22 \%$ habían compartido agujas y el $59 \%$ habían compartido material de inyección, a pesar de que la mayoría (96\%) conocían los riesgos.

Un estudio en Australia sobre las infecciones de transmisión sexual entre las personas que ejercen y sus clientes y sobre las diferencias en la prevalencia en función de los tipos de prostitución ${ }^{7}$. En el estudio fueron analizadas 247 personas que ejercían la prostitución y completaron cuestionarios anónimos con edades entre los 19 y 57 años y 185 clientes de entre los 19 a 72 años. Los resultados fueron los siguientes: hubo poca variación en la prevalencia de las ITS en función de los tipos de prostitución. Sin embargo, los clientes que solían acceder a los servicios ilegales en la calle registraron un mayor número de enfermedades que los clientes que acudían a pisos o club legalizados. Esto sugiere que el riesgo de infección no es similar en todos los tipos de prostitución.

Un estudio longitudinal que exploraba la salud en un período de 15 años (1986 a 1993) entre las mujeres que ejercían en Londres ${ }^{8}$. Se obtuvieron datos de los resultados en $130(37 \%)$ de las 354 mujeres que se intentaron analizar. Se evaluaron las siguientes variables: el estado vital, la ocupación más reciente, la duración del trabajo sexual, las infecciones de transmisión sexual y problemas de salud. Los resultados fueron los siguientes: la mayoría (el 59\%) se encontraban todavía en la prostitución y llevaban ejerciendo una media de 13,6 años. Hubo seis muertes, una mortalidad de 4,8 por cada 1.000 personas. Las mujeres sobrevivientes tuvieron un riesgo acumulado alto (el $93 \%$ ) de ITS. Los problemas de salud mental afectaban al $40 \%$ y los de la adicción al $64 \%$. Se encontraron diferencias significativas en los resultados de salud entre las mujeres que todavía estaban en la prostitución y las que lo habían dejado. Hubo un alto nivel de

6 Nikki Jeal y Chris Salisbury, "A health needs assessment of street-based prostitutes: cross-sectional survey", Journal of Public Health 26 (2004): 147-151, https://doi.org/10.1093/pubmed/fdh124

7 Charlotte Seib et al., "Sexually transmissible infections among sex workers and their clients: variation in prevalence between sectors of the industry", Sex Health 6 (2009): 45-50, https://www.ncbi.nlm.nih.gov/pubmed/19254491

8 Helen Ward y Susan Day, "What happens to women who sell sex? Report of a unique occupational Cohort", Sexually Transmitted Infections 82 (2006): 413-417, https://doi.org/10.1136/sti.2006.020982 
movilidad laboral, y 31 mujeres (el $37 \%$ ) habían terminado la formación profesional o estudios superiores, incluidos el nivel de postgrado.

Otros autores sugieren un vínculo complejo entre el uso de drogas ilegales y la subsiguiente entrada en prostitución ${ }^{9}$, ya que encontraron que un $66 \%$ de las prostitutas entrevistadas habían usado drogas antes de comenzar a ejercer la prostitución, un 18\% comenzaron ambas conductas al mismo tiempo y un $17 \%$ usó drogas después de comenzar a ejercer la prostitución. Aunque es necesaria una demostración empírica para establecer la relación causal entre estos sucesos (uso de drogas y entrada en prostitución), los datos existentes demuestran la fuerte secuencia temporal de estos eventos críticos ya que el abuso de sustancias suele preceder a la entrada en prostitución.

El consumo de drogas durante el ejercicio de la prostitución fue analizado por Brewis and Linstead ${ }^{10}$. Para estos autores las drogas pueden ser usadas por múltiples motivos en el mundo de la prostitución: algunas veces para mantener a las trabajadoras despiertas durante largos días y largas noches, algunas ocasiones para relajarlas y aliviar la sensación de lo poco placentero que es el trabajo, otras veces porque ayuda a jugar el papel requerido en el trabajo y para llegar a ser el producto que los clientes demandan.

Los problemas emocionales en las vidas de las mujeres que ejercen la prostitución son menos visibles y por lo tanto menos estudiados. Los estudios se centran más en los aspectos sensacionalistas (violencia, tráfico de mujeres, enfermedades, problemas de salud, delincuencia, etc.) que en aspectos relacionados con el bienestar de su vida emocional.

En relación a la tensión emocional o estrés derivado de las condiciones en las que se desarrolla la prostitución Vanwesenbeeck ${ }^{11}$ analiza el síndrome de Burnout de las mujeres $(\mathrm{N}=96)$ que ejercen en pisos o club en los Países Bajos. Los niveles de desgaste profesional se analizaron en 3 dimensiones: agotamiento emocional, despersonalización y competencia personal. La muestra fue comparada con mujeres enfermeras y con pacientes que tenían problemas psicológicos relacionados con el trabajo. Los resultados son los siguientes: únicamente la dimensión despersonalización fue significativamente más alta en las mujeres que ejercían en comparación con las enfermeras y muy similar al grupo de pacientes que tenían problemas psicológicos relacionados con el trabajo. El $42 \%$ de la varianza en la despersonalización se explica por no trabajar de forma autónoma, por las reacciones negativas sociales, las experiencias de violencia y la falta de control en la interacción con los clientes. La despersonalización podría ser una estrategia para hacer frente a las condiciones negativas y experiencias en el trabajo; sin embargo, estaba relacionada con los indicadores de estrés y agotamiento emocional. Más de la mitad (53\%) de la varianza en el agotamiento emocional se explica por la falta de apoyos en el desarrollo del trabajo, las reacciones sociales

9 John Potterat et al., "Pathways to prostitution: The chronology of sexual and drug abuse milestones", The Journal of Sex Research 35 (1998): 333-240, https://doi.org/10.1080/00224499809551951.

10 Joanna Brewis and Stephen Linstead, "The worst thing is the screwing: Consumption and the management of identity in sex work", Gender, work and organization 7 (2002): 84-97, https://doi.org/10.1111/1468$\underline{0432.00096}$

11 Ine Vanwesenbeeck, "Burnout among female indoor sex workers", Archives of Sexual Behavior 34 (2005): 627-639, https://link.springer.com/article/10.1007/s10508-005-7912-y 
negativas, el no ser un trabajo que se elige de forma libre y la falta de motivación o motivación negativa hacia el trabajo. La competencia personal fue mayor entre las mujeres que ejercían y que tenían una actitud profesional positiva, que comenzaron el trabajo sexual a una edad relativamente madura y que se encontraban bien con el apoyo de sus colegas y con la gestión de la actividad. Se concluye que el desgaste no está tan relacionado con el trabajo sexual en sí, sino con el trabajo sexual en determinadas condiciones, como son las reacciones negativas o el estigma social y las experiencias relacionadas con el estigma: la violencia, la falta de apoyos para la gestión, la falta de identidad como trabajadora, etc.

En otro estudio se comparan la salud mental y física, las agresiones y las redes sociales de las mujeres que ejercen en Nueva Zelanda con una muestra de control (población normal) ${ }^{12}$. La muestra estuvo compuesta por 29 mujeres que ejercían en diferentes modalidades. Se les aplicó el Cuestionario de Salud General (GHQ-28) y otros sobre abusos sexuales y autoestima. Los resultados son los siguientes: no hubo diferencias en la salud mental en el GHQ-28 o en la autoestima entre los dos grupos. No hubo diferencias en la evaluación de su salud física o la calidad de sus redes sociales. En el GHQ-28 la puntuación media para el grupo de mujeres que ejercían fue de $5,3 \%$, cuya diferencia no es estadísticamente significativa con el grupo de control (3,5\%). Las conclusiones para estos autores son las siguientes: no se han encontrado pruebas de que la prostitución (per se) esté ligada a un aumento de la morbilidad psiquiátrica, aunque puede haber grupos de mujeres con problemas específicos al igual que en la población normal; la mayoría de las mujeres que ejercían $(90 \%)$ se sentían bien al igual que el grupo de control; el $90 \%$ de las mujeres que ejercían reconocían que su autoestima había aumentado en los últimos 5 años. Los datos no proporcionan ninguna prueba convincente de que las mujeres que ejercen la prostitución tengan más problemas de salud física o psicológica, baja autoestima o alteración de las relaciones sociales que la población general; a pesar de los problemas personales derivados del ejercicio (más riesgos de sufrir agresiones físicas, abusos sexuales, el estigma, etc.) estas mujeres tienen relaciones adecuadas con sus parejas, amigos y compañeras.

Los estudios más recientes publicados en España sobre aspectos psicológicos y a través de aplicación de pruebas estandarizadas son los siguientes:

El estudio de Pinedo ${ }^{13}$ que aplica los cuestionarios de autoestima Rosenberg Self-Esteem (RSE) y de depresión y ansiedad SCL-90-R. La muestra está compuesta por 20 mujeres, 9 de ellas ejercen prostitución en club o piso y 11 en la calle de Valladolid. Las puntuaciones en depresión y ansiedad son altas en ambas escalas para ambos grupos. En depresión, para el grupo de mujeres de la calle la media en centiles es de $95,09 \%$ y en las mujeres de club o piso es de $93,89 \%$. En ansiedad, para el grupo de mujeres de la calle la media en centiles es de 93,91\% y en las mujeres de club o piso es de $76,56 \%$. No se han encontrado diferencias significativas entre las medias de los dos grupos ni en depresión ni en ansiedad. En cuanto a las puntuaciones en autoestima se han encontrado diferencias significativas entre ambos grupos, de manera que el grupo de mujeres que ejercen

12 Sarah Romans et al., "The mental and physical health of female sex workers: a comparative study", Australian and New Zealand Journal of Psychiatry 35 (2001): 75-80, https://doi.org/10.1046/j.1440-1614.2001.00857.x.

13 Ruth Pinedo, "Calidad de vida y relaciones interpersonales de las mujeres que ejercen prostitución" (trabajo de suficiencia investigadora no publicado, Universidad de Salamanca, 2005). 
en club o piso tienen mejor puntuación en esta escala que las que ejercen en la calle.

Otro estudio similar al anterior investiga los factores de estrés emocional en la $v \operatorname{vid}^{14}$. Se realizan entrevistas semi-estructuradas a 68 mujeres que ejercen en entornos diferentes. Los autores y, por lo tanto, la investigación, parten de la idea de que las vidas de las mujeres que ejercen son muy similares a las vidas de muchas mujeres que trabajan en otras ocupaciones. A las mujeres de este estudio les producía estrés la falta de control sobre las condiciones de trabajo, las relaciones difíciles con otras mujeres en el trabajo, compaginar su vida privada (el hogar, los hijos, la pareja), las relaciones con la familia y los amigos, etc. Aunque existen algunas similitudes, las mujeres que ejercen tienen que hacer frente al estigma y sus consecuencias, el ocultamiento de su actividad, incluso si ya la han abandonado, los peligros y riesgos en cuanto a su salud y a su seguridad personal, la ilegalidad o no regularización en la que se desarrolla la actividad, etc.

\section{Prostitución y arte}

En 2015, el Ministerio de Cultura francés llevó a cabo una exitosa exposición en el Museo Orsay: "Esplendor y miseria de la prostitución en Paris, 1850-1910" 15. Comisariada por Marie Robert e Isolde Pludemacher ${ }^{16}$ y presentada por Richard Thomson, profesor de Historia del Arte de la Universidad de Edimburgo. La exposición presentaba más de doscientas obras de grandes maestros que han tratado el tema desde diversos ángulos. Las obras contenían desde el clásico "Olympia" de Manet ${ }^{17}$ a "Las señoritas de Avignon" de Picasso e ilustraciones sobre el tema de Bernard, Degas, Renoir, Toulouse-Lautrec ${ }^{18}$. En un intento de hacer la exposición más atractiva, se completo con objetos históricos relacionados como un sillón de amor, donde el Príncipe de Gales, Eduardo VII, retozaba con dos acompañantes mientras esperaba su llegada al trono de Inglaterra y la reproducción de un salón y la habitación de una profesional que tenía la más alta cotización. La

14 Lois A. Jackson et al., "Stress in the sex trade and beyond: Women working in the sex trade talk about the emotional stressors in their working and home lives", Critical Publica Health 17 (2007): 257-271, http://dx.doi.org/10.1080/09581590701549535.

15 Alex Vicente, "El oficio más antiguo", El País Semanal, 27 de octubre de 2015, https://elpais.com/elpais/2015/10/23/eps/1445594658_483842.html. En 2015, el Ministerio de Cultura francés llevó a cabo una exitosa exposición en el Museo Orsay: Esplendor y miseria de la prostitución en Paris, 1850-1910. En la inauguración se batió un record de visitantes, 7000 asistentes. Se puede obtener información al respecto en la prensa internacional, y en nuestro país se puede consultar el artículo de Alex Vicente publicado en El País Semanal.

16 Isolde Pludemacher, comisaria del museo parisiense, justificó la motivación y el título de la exposición a la prensa internacional con los siguientes comentarios: "la prostituta ha sido un personaje ignorado por la Historia del Arte pese a haber sido una figura central de la pintura del siglo XIX. Existió entonces una identificación entre el artista y la meretriz fruto de su apego por la vida bohemia" y añade: "La exposición revela que París se convirtió, en tiempos del segundo imperio y la belle époque, en capital europea del sexo remunerado. Los artistas reprodujeron un fenómeno que se extendía por bistrós y bulevares, convirtiendo la frenética actividad de esquineras en el tema moderno por definición".

17 La Olympia que retrató Manet fue también musa de Van Gogh, era Agostina Segatori. Van Gogh la retrató en su famoso cuadro Mujer en el Café de Tambourini, y es con quien se especula que mantuvo relaciones en 1887. Lettres 461-462: Vincent Van Gogh, et al., Correspondance complète de Vincent Van Gogh: enrichie de tous les dessins originaux (Paris: Gallimard, 1960), Lettres 459-662, 1886-1890.

18 Toulouse-Lautrec pintó el conocido retrato de Van Gogh en el ya referido Café Tambourini. 
reconocida Olympia de Manet estuvo acompañada de una colección de fotografías y películas pornográficas de la época. En la exposición también hubo protagonismo para una española de armas tomar, la legendaria gallega conocida como "La Bella Otero" con una lista de amantes monárquicos tan notables como Guillermo II de Alemania, Nicolás II de Rusia, Leopoldo II de Bélgica. Agustina del Carmen Otero Iglesias, más conocida como Carolina Otero, fue retratada por el histórico fotógrafo Leopold-Emilie Reutlinger, retratista de celebridades y creador de tarjetas postales bestseller, considerado uno de los pioneros de la fotografía erótica que tuvo entre sus clientes a Mata Hari, Colette y una larga lista de las más brillantes celebridades de la época.

Toda esta información recogida en el entorno artístico ha sido la base para la elaboración de la terapia psicológica cognitiva diseñada para unificar al proceso de Psicoballet, con el fin de elevar la autoestima y ayudar a superar los posibles traumas y procesos de ansiedad implicados en estas experiencias y constatados en las fases de trabajo realizadas con este colectivo. La historia del arte y las obras pictóricas y escultóricas relacionadas con el tema de la prostitución son la base para los procesos reflexivos, informativos y el cambio de actitudes para reiniciar un nuevo proceso vital. También hemos utilizado como fuente de inspiración los trabajos de la activista, modelo y artista Lauren Zoe ${ }^{19}$.

Zoe en su etapa de modelo tuvo experiencias muy negativas, fue asaltada sexualmente por un artista y al denunciarlo a la policía le dijeron que no podían hacer nada al respecto porque ella estaba desnuda. Esta historia parece no ser un caso aislado ni anecdótico, en la industria del sexo ocurren situaciones similares, según Zoe, en numerosas declaraciones en los medios de comunicación internacionales, existen estructuras de género y otras relativas a la sexualidad que están "enquistadas". A raíz de sus propias experiencias creó los talleres, denominados "porn life drawing" (dibujo pornográfico del natural) que consiste en una nueva modalidad de taller de arte cuyas modelos son trabajadoras sexuales con un propósito: demostrar que el hecho de que estés siendo sexual no otorga ningún derecho a nadie a creerse que puede serlo contigo, de este modo ha vuelto a convertir a trabajadoras sexuales en musas de artistas. El sentido de estas sesiones, que impulsa desde "Sex Worker Art Collective", no es "empoderar" -palabra muy de actualidad- a las trabajadoras sexuales, sino humanizar a las mujeres que han hecho de su cuerpo un "modus vivendi" y crear puentes con un público de artistas y consumidores de arte, desmitificando la industria del sexo. Sex Worker Art Collective es un grupo de trabajadoras sexuales, activistas por los derechos de las trabajadoras sexuales y aliadas de las trabajadoras sexuales que crean eventos comunitarios para estimular la conversación sobre la desestigmatización de las trabajadoras sexuales. El colectivo ha abierto en el Sur de Londres un espacio público comunitario, junto a una galería pública, donde se muestra arte para comunicar visualmente la política en torno a los derechos de las trabajadoras sexuales, los derechos laborales, el clasismo y el sexismo. El colectivo es anfitrión de eventos artísticos utilizados como un espacio sin prejuicios para educar y como

19 Beatriz García, "Las prostitutas vuelven a ocupar un lugar en el mundo del Arte", El Confidencial, 9 de mayo de 2016. "Sex Worker Art Collective": Para poder conocer algunas imágenes de las acciones de este colectivo y del Sistema Zoe se puede consultar el artículo que Beatriz García le dedicó al tema en el diario El Confidencial. 
un espacio social y teatral. Este colectivo surgió de Goldsmiths, de la Universidad de Londres, de la Goldsmiths Sex Worker Solidarity Society, que es un grupo de apoyo para trabajadores sexuales en el campus. Sus experiencias son relevantes y han supuesto un importante bastión en las intervenciones sociales directas con el ámbito de la prostitución, al margen de morbos y polémicas.

La Historia del Arte permite abordar el tema de la prostitución desde una perspectiva liberadora de estigmas y problemas psicológico-sociales añadidos que facilita la recuperación voluntaria y la libertad de cambio de conciencia.

La aproximación artística, como herramienta de intervención, junto con los revolucionarios trabajos y experiencias desarrolladas en $\mathrm{Cuba}^{20}$ con la introducción de las artes en la aplicación clínica y la intervención social ${ }^{21}$, específicamente a través del ballet ${ }^{22}$ y los trabajos de adaptación y validación previos realizados en España desde el Instituto Universitario de Danza Alicia Alonso, de la Universidad Rey Juan $\mathrm{Carlos}^{23}$, en diferentes aplicaciones clínicas con pacientes con enfermedades psiquiátricas, neurológicas y psicológicas, así como con víctimas de violencia de género, explotación y violencia sexual, nos decidieron a afrontar proyectos de intervención social con este colectivo y su problemática desde una perspectiva arteterapéutica ${ }^{24}$ lo que nos ha permitido comprobar las utilidades y beneficios terapéuticos que aportan las artes al ámbito de la clínica y la educación y las grandes ventajas que tiene toda actividad relacionada con el Patrimonio Artístico y Museístico en los colectivos desfavorecidos.

\section{Metodología}

\subsection{Sujetos}

En el estudio participaron 34 mujeres que ejercían la prostitución, con edades comprendidas entre los 19 y los 41 años de edad (media de edad de 26,7 años). La mayoría eran inmigrantes y víctimas de explotación sexual.

20 Georgina Fariñas, Temas de Psicoballet, (La Habana: Hospital Psiquiátrico de La Habana, 1999).

21 Georgina Fariñas, Psicoballet, teoría y práctica en Cuba y Puerto Rico, (Puerto Rico: Universidad de Puerto Rico, 2004).

22 Trabajos desarrollados en la década de los años setenta del siglo XX por la síntesis creada por la Prima Ballerina Assoluta, Alicia Alonso, directora, coreógrafa y fundadora de una de las más prestigiosas compañías del mundo "El Ballet Nacional de Cuba", junto con el histórico y legendario director del Hospital Psiquiátrico de la Habana, el Dr. Eduardo Bernabé Ordaz Ducunge y la prestigiosa psicóloga cubana Georgina María Fariñas García. Una aproximación validada por la investigación experimental y la experiencia reconocida por la UNESCO en 1984, después de analizar un dossier de 29.000 casos tratados con éxito mediante el Psicoballet.

23 Amador Cernuda, "Arte social comunitario y aplicaciones clínicas del psicoballet. 40 años de experiencia cubana”, en Congreso Internacional de Intervención Social, Arte Social y Arteterapia, 28 de noviembre-1 de diciembre de 2012, Murcia, consultado el 28 de marzo de 2019, http://congresos.um.es/isasat/isasat2/paper/view/28031

24 Amador Cernuda, "The Arts in Clinical Health Programs for the Recovery of Diseases and to Improve Quality of Life", en Case Study of Innovative Projects-Successful Real Cases, ed. Bernardo Llamas Moya, María Dolores Storch de Gracia y Luis F. Mazadiego (London: Intech, 2017), 263-278, http://dx.doi.org/10.5772/intechopen.69344 


\subsection{Instrumentos}

A todas se les realizó una entrevista clínica y se les aplicó el test STAI de Ansiedad de Spielberger, la Escala de Autoestima de Rosemberg, el MSCEIT (Test de Inteligencia Emocional Mayer-Salovey-Caruso) y el MMPI-2-RF (Inventario Multifásico de Personalidad de Minnesota-2 Reestructurado) de Y.S. Ben-Porath y A. Tellegen, para valorar los efectos de las sesiones de psicoballet, de realidad virtual y los efectos de las visitas. En este estudio utilizamos la adaptación al castellano del Profile of Mood States (POMS) que se realizó en la Universidad Autónoma de Madrid por Cernuda ${ }^{25}$ en 1985.

El cuestionario Profile of Mood States (POMS) es un instrumento de valoración del estado emocional desarrollado por McNair, Loor and Droppleman ${ }^{26}$ para población no psiquiátrica y psiquiátrica externa. El POMS es un instrumento multidimensional con una concepción del estado de ánimo influenciada por el marco teórico de Kurt Lewin que considera a la persona como parte de un todo dentro de un sistema dinámico. Su objetivo principal es evaluar los estados de ánimo o estados afectivos transitorios fluctuantes.

Basado en lo anteriormente expuesto el presente estudio se propone analizar las posibles diferencias en las puntuaciones de las participantes antes y después de las sesiones de psicoballet a través del POMS. El POMS incluye 58 acápites agrupados en seis factores: tensión, depresión, cólera, vigor, fatiga, confusión. El vigor se refiere a un estado de ánimo positivo y los demás factores a un estado de ánimo negativo. Cada acápite es valorado siguiendo un formato tipo Likert, con cinco alternativas de respuesta: Nada (0), Poco (uno), Regular (dos), Bastante (tres), Mucho (cuatro). Todos los acápites están formulados en la misma dirección, menos eficiente (confusión) y relajado (tensión).

\subsection{Procedimiento}

Las 34 participantes se entrevistaron clínicamente y se evaluaron con test psicológicos para determinar su estado al inicio del tratamiento, así como su evolución y estado al finalizar el mismo. El proceso duro seis meses con 48 sesiones de psicoballet y arteterapia escénica, 8 visitas guiadas a museos de arte y etnología y 24 sesiones de nuevas tecnologías de apoyo a la psicoterapia cognitiva y a la sensibilización artística patrimonial mediante la aplicación de la realidad virtual.

\section{Resultados}

Se analizaron los datos con el SPSS v.19 para Windows. Los resultados se muestran en la Tabla 1, Tabla 2 y Tabla 3.

25 Amador Cernuda, "Medida del Perfil de Estados de Humor", en Manual de Prácticas de Psicología General, ed. José Luis González (Madrid: Universidad Autónoma de Madrid, 1985), 17-20.

26 Douglas Mcnair, Maurice Loor y Leo F. Droppleman, Manual for the Profile of Mood States (San Diego: Educational and Industrial Testing Services, 1971). 
Tabla 1. Prueba T. Estadísticos de muestras relacionadas

\begin{tabular}{|c|c|c|c|c|c|}
\hline & & Media & $\mathbf{N}$ & $\begin{array}{c}\text { Desviación } \\
\text { típica }\end{array}$ & $\begin{array}{l}\text { Error típico } \\
\text { de la media }\end{array}$ \\
\hline \multirow{2}{*}{ Par 1} & Tensión antes & 56,1 & 34 & 5,656 & 1,031 \\
\hline & Tensión después & 52,45 & 34 & 5,361 & 0,995 \\
\hline \multirow{2}{*}{ Par 2} & Depresión antes & 51,91 & 34 & 4,397 & 0,87 \\
\hline & Depresión después & 48,87 & 34 & 3,629 & 0,647 \\
\hline \multirow{2}{*}{ Par 3} & Angustia antes & 52,47 & 34 & 5,03 & 0,911 \\
\hline & Angustia después & 49,67 & 34 & 4,611 & 0,841 \\
\hline \multirow{2}{*}{ Par 4} & Vigor antes & 51,4 & 34 & 3,597 & 0,656 \\
\hline & Vigor después & 53,2 & 34 & 4,231 & 0,771 \\
\hline \multirow{2}{*}{ Par 5} & Fatiga antes & 50,1 & 34 & 3,863 & 0,703 \\
\hline & Fatiga después & 47,77 & 34 & 3,546 & 0,685 \\
\hline \multirow{2}{*}{ Par 6} & Confusión antes & 50,13 & 34 & 3,64 & 0,671 \\
\hline & Confusión después & 47,26 & 34 & 3,429 & 0,628 \\
\hline
\end{tabular}

Tabla 1: Tabla elaborada con los datos de las medias obtenidas en las diferentes sesiones. (SPSS v.19 para Windows). Fuente: autor.

Diferencia de medias: los resultados indican que la ansiedad media de las participantes desciende un $40 \%$ después del proceso de tratamiento y la autoestima se eleva como media global un 35\% desarrollando capacidades de afrontamiento y comunicación.

Existen diferencias significativas en las medidas tomadas antes y después de las sesiones de psicoballet en todas las variables medidas por el POMS: Tensión, Depresión, Angustia, Vigor, Fatiga y Confusión ( $\mathrm{p}<0,01)$.

Las participantes presentan mayores puntuaciones en Tensión, Depresión, Angustia, Fatiga y Confusión antes de la sesión de psicoballet y una mayor puntuación en Vigor después de la sesión de psicoballet ${ }^{27}$.

Según se van sucediendo las sesiones los datos son más contundentes.

\section{Discusión}

Nos encontramos ante un colectivo con enormes necesidades de atención, físicas y psicológicas que necesita más investigación desprovista de prejuicios porque ante todo son personas con una situación de marginalidad que, en un porcentaje

27 W.P. Morgan y R.W. Johnson, "Psychological characterization of the elite wrestler: A mental health model", Medicine and Science in Sports 9 (1977): 55-56. Es interesante acentuar que las participantes que muestran en las medidas del POMS todas las variables de los estados de humor por debajo de la media, excepto el factor Vigor que estaría por encima, manifiestan el conocido perfil iceberg que sería, en esencia, el perfil de una persona psicológicamente sana y el POMS se revela como la prueba más efectiva para el propósito de predicción del éxito y el fracaso. El perfil iceberg evidencia el efecto positivo del proceso de intervención artística en la evolución y recuperación de la mayoría de las participantes. 
mayoritario, ejercen estas actividades por presión, manipulación de su realidad y por intereses ajenos ilícitos que se enriquecen de su actividad. Estamos ante un fenómeno vergonzante, no por la actividad en sí, sino por lo que refleja, en muchos casos un auténtico mantenimiento de la esclavitud en la época de los derechos humanos.

Tabla 2. Prueba T. Correlaciones de muestras relacionadas

\begin{tabular}{llllc}
\hline & & N & Correlación & Sig. \\
\hline Par 1 & Tensión antes y Tensión después & 34 & 0,929 & 0 \\
Par 2 & Depresión antes y Depresión después & 34 & 0,923 & 0 \\
Par 3 & Angustia antes y Angustia después & 34 & 0,859 & 0 \\
Par 4 & Vigor antes y Vigor después & 34 & 0,779 & 0 \\
Par 5 & Fatiga antes y Fatiga después & 34 & 0,935 & 0 \\
Par 6 & Confusión antes y Confusión después & 34 & 0,874 & 0 \\
\hline
\end{tabular}

Tabla 2: Tabla elaborada con los datos de las medias obtenidas en las diferentes sesiones.

(SPSS v.19 para Windows). Fuente: autor.

Tabla 3. Prueba T. Prueba de muestras relacionadas

\begin{tabular}{|c|c|c|c|c|c|c|c|c|}
\hline & \multirow[b]{2}{*}{ Media } & \multirow{2}{*}{$\begin{array}{c}\text { Desviación } \\
\text { típ. }\end{array}$} & \multirow{2}{*}{$\begin{array}{l}\text { Error } \\
\text { típ. de la } \\
\text { media }\end{array}$} & \multicolumn{2}{|c|}{$\begin{array}{c}\text { 95\% Intervalo de } \\
\text { confianza para la } \\
\text { diferencia }\end{array}$} & \multirow[b]{2}{*}{$\mathbf{t}$} & \multirow[b]{2}{*}{ Gl } & \multirow{2}{*}{$\begin{array}{l}\text { Sig. } \\
\text { (bila- } \\
\text { (teral) }\end{array}$} \\
\hline & & & & Inferior & Superior & & & \\
\hline \multicolumn{9}{|l|}{ Part 1} \\
\hline $\begin{array}{l}\text { Tensión antes } \\
\text { Tensión después }\end{array}$ & - & 2,034 & 0,371 & 2,24 & 3,76 & 8,078 & 33 & 0 \\
\hline \multicolumn{9}{|l|}{ Part 2} \\
\hline $\begin{array}{l}\text { Depresión antes } \\
\text { Depresión } \\
\text { después }\end{array}$ & 2,767 & 1,942 & 0,355 & 2,042 & 3,492 & 7,803 & 33 & 0 \\
\hline \multicolumn{9}{|l|}{ Part 3} \\
\hline $\begin{array}{l}\text { Angustia antes } \\
\text { Angustia después }\end{array}$ & 2,6 & 2,568 & 0,469 & 1,641 & 3,559 & 5,546 & 33 & 0 \\
\hline \multicolumn{9}{|l|}{ Part 4} \\
\hline $\begin{array}{l}\text { Vigor antes } \\
\text { Vigor después } \\
\end{array}$ & 1,8 & 2,644 & 0,483 & $-2,787$ & $-0,813$ & 3,728 & 33 & 0,001 \\
\hline \multicolumn{9}{|l|}{ Part 5} \\
\hline $\begin{array}{l}\text { Fatiga antes } \\
\text { Fatiga después } \\
\end{array}$ & 2,333 & 1,47 & 0,268 & 1,784 & 2,882 & 8,694 & 33 & 0 \\
\hline \multicolumn{9}{|l|}{ Part 6} \\
\hline $\begin{array}{l}\text { Confusión antes } \\
\text { Confusión } \\
\text { después }\end{array}$ & 2,667 & 1,788 & 0,326 & 1,999 & 3,334 & 8,171 & 33 & 0 \\
\hline
\end{tabular}

Tabla 3: Tabla elaborada con los datos de las medias obtenidas en las diferentes sesiones

(SPSS v.19 para Windows). Fuente: autor. 
Es necesario establecer programas educativos que cambien la visión poblacional y terminar con las numerosas posiciones de hipocresía social y política que se mantienen ante esta temática. Ante todo facilitar políticas de fomento de la salud física y mental, así como, la salud sexual y reproductiva, informando y facilitando medios y conocimiento, teniendo en cuenta que el perfil educativo de la mayoría es más bien bajo -aunque nos encontremos con una parte de la población que ejerce que tiene estudios superiores, lo habitual es una gran ignorancia- lo que facilita la explotación y pone en riesgo su salud y sus vidas en la búsqueda de soluciones a sus problemáticas diarias.

Los estudios mayoritarios son de problemáticas relacionadas con el VIH/SIDA y en general las infecciones de transmisión sexual, sin embargo, los estudios en salud mental son minoritarios y estimamos que son muy importantes porque la depresión, la ansiedad y las ideaciones suicidas son muy numerosas y tienen implicaciones preocupantes.

\section{Conclusiones}

Existe una nítida relación entre abuso de sustancias y ejercicio de la prostitución. Las sustancias más consumidas en la población estudiada son alcohol, cocaína y cannabis. Existe un porcentaje muy alto de presencia de abusos sexuales en la infancia, abuso de sustancias y ejercicio de la prostitución. El nivel de ansiedad es elevado, los síntomas depresivos y la ideación suicida son muy habituales, estando sometidas a un ambiente de estrés continuo, estrés que les produce miedo e inseguridad, fundamentalmente.

La utilización del patrimonio museístico y artístico para la reflexión y realización de la psicoterapia cognitiva ha sido muy positivo, útil y humanizante, permitiendo adoptar por parte de las participantes actitudes favorecedoras del cambio.

Las prácticas artísticas integradas del psicoballet han facilitado la comunicación. Al trabajar juntas con los mismos problemas y la movilización del cuerpo han permitido romper estructuras rígidas corporales y bloqueos, facilitando la liberación de la tensión y la reducción de la ansiedad. El Arte es un coadyuvante en los procesos terapéuticos y de integración social que debe ser más tenido en cuenta en futuros proyectos y debe tener un mayor desarrollo en nuestro país siguiendo las experiencias de países más avanzados y con mayor experiencia, como Estados Unidos, Canadá, Israel, Japón y Reino Unido.

\section{Fuentes y referencias bibliográficas}

Brewis, Joanna, y Stephen Linstead. "The worst thing is the screwing: Consumption and the management of identity in sex work". Gender, work and organization 7 (2002): 8497. https://doi.org/10.1111/1468-0432.00096

Cernuda Lago, Amador. "Medida del Perfil de Estados de Humor". En Manual de Prácticas de Psicología General, editado por José Luis González, 17-20. Madrid: Universidad Autónoma de Madrid, 1985. 
Cernuda Lago, Amador. “Arte social comunitario y aplicaciones clínicas del psicoballet. 40 años de experiencia cubana". En Congreso Internacional de Intervención Social, Arte Social y Arteterapia. 28 de noviembre-1 de diciembre de 2012, Murcia. http://congresos.um.es/isasat/isasat2/paper/view/28031

Cernuda Lago, Amador. "Efectos de un programa de rehabilitación psicosocial y educativa mediante actividad física y artística con un grupo de víctimas de explotación sexual”. En III Congreso Internacional Multidisciplinar de Investigación Educativa-CIMIE14. 3-4 de julio de 2014, Segovia. http://amieedu.org/actascimie14/?cat=18

Cernuda, Amador. "The Arts in Clinical Health Programs for the Recovery of Diseases and to Improve Quality of Life". En Case Study of Innovative Projects-Successful Real Cases, editado por Bernardo Llamas Moya, María Dolores Storch de Gracia, y Luis F. Mazadiego, 263-278. London: Intech, 2017. http://dx.doi.org/10.5772/intechopen.69344

Fariñas, Georgina. Temas de Psicoballet. La Habana: Hospital Psiquiátrico de La Habana, 1999.

Fariñas, Georgina. Psicoballet, teoría y práctica en Cuba y Puerto Rico. Puerto Rico: Universidad de Puerto Rico, 2004.

García, Beatriz. "Las prostitutas vuelven a ocupar un lugar en el mundo del arte". El Confidencial, 9 de mayo de 2016. https://www.elconfidencial.com/cultura/2016-0509/arte-dibujo-del-natural-erotismo-sexo_1196770

Jackson, Lois A., Carolyn G. Bennett, y Barbara A. Sowinski. "Stress in the sex trade and beyond: Women working in the sex trade talk about the emotional stressors in their working and home lives". Critical Publica Health 17 (2007): 257-271. http://dx.doi.org/10.1080/09581590701549535

Jeal, Nikki., and Chris Salisbury. "A health needs assessment of street-based prostitutes: cross-sectional survey". Journal of Public Health 26 (2004): 147-151. https://doi.org/10.1093/pubmed/fdh124

Mcnair Douglas, Maurice Loor, and Leo F. Droppleman. Manual for the Profile of Mood States. San Diego: Educational and Industrial Testing Services; 1971.

Pinedo González, Ruth. "Calidad de vida y relaciones interpersonales de las mujeres que ejercen prostitución”. Trabajo de suficiencia investigadora no publicado. Universidad de Salamanca, 2005.

Potterat, John J., Richard B. Rothenberg, Stephen Q. Muth, William W. Darrow, and Lynanne Phillips-Plummer. "Pathways to prostitution: The chronology of sexual and drug abuse milestones". The Journal of Sex Research 35 (1998): 333-240. https://doi.org/10.1080/00224499809551951

Romans, Sarah. E., Kathleen Potter, Judy Martín, and Peter Herbison. "The mental and physical health of female sex workers: a comparative study". Australian and New Zealand Journal of Psychiatry 35 (2001): 75-80. https://doi.org/10.1046/j.14401614.2001.00857.x

Seib, Charrlotte, Joseph Debattish, Jane Anne Fischer, and Michael Dume. "Sexually transmissible infections among sex workers and their clients: variation in prevalence between sectors of the industry". Sex Health 6 (2009): 45-50. https://www.ncbi.nlm.nih.gov/pubmed/19254491

Van Gogh, Vincent, Maurice Beerblock, Louis Roëlandt, and Georges Charensol. Correspondance complète de Vincent Van Gogh: enrichie de tous les dessins originaux. Paris: Gallimard, 1960.

Vanwesenbeeck, Ine. "Another decade of social scientific work on sex work: A review of research 1990-2000". Annual Review of Sex Research 12 (2001): 242-289. 
https://www.ncbi.nlm.nih.gov/pubmed/12666742

Vanwesenbeeck, Ine. "Burnout among female indoor sex workers". Archives of Sexual Behavior 34 (2005): 627-639. https://link.springer.com/article/10.1007/s10508-0057912-y

Vicente, Álex. "El oficio más antiguo". El País Semanal, 27 de octubre de 2015. https://elpais.com/elpais/2015/10/23/eps/1445594658_483842.html

Ward, Helen and Susan Day. "What happens to women who sell sex? Report of a unique occupational Cohort". Sexually Transmitted Infections 82 (2006): 413-417. https://doi.org/10.1136/sti.2006.020982 